\title{
BMJ Open Glomerular filtration rate (GFR) during and after STEMI: a single-centre, methodological study comparing estimated and measured GFR
}

\author{
Dimitrios Venetsanos, Joakim Alfredsson, Mårten Segelmark, Eva Swahn, \\ Sofia Sederholm Lawesson
}

To cite: Venetsanos $D$, Alfredsson J, Segelmark M, et al. Glomerular filtration rate (GFR) during and after STEMI: a single-centre, methodological study comparing estimated and measured GFR. BMJ Open 2015; : $: 007835$.

doi:10.1136/bmjopen-2015007835

- Prepublication history and additional material is available. To view please visit the journal (http://dx.doi.org/ 10.1136/bmjopen-2015007835).

Received 9 February 2015 Revised 1 July 2015 Accepted 15 July 2015

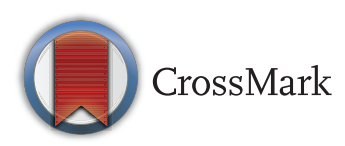

Division of Cardiovascular Medicine, Department of Medical and Health Sciences, Linköping University, Linköping, Sweden

Correspondence to Dr Dimitrios Venetsanos; dimitrios.venetsanos@liu.se

\section{ABSTRACT}

Objectives: To validate the performance of the most commonly used formulas for estimation of glomerular filtration rate (GFR) against measured GFR during the index hospitalisation for ST-elevation myocardial infarction (STEMI).

Setting: Single centre, methodological study. Participants: 40 patients with percutaneous coronary intervention-treated STEMI were included between November 2011 and February 2013. Patients on dialysis, cardiogenic shock or known allergy to iodine were excluded.

Outcome measures: Creatinine and cystatin $C$ were determined at admission and before discharge in 40 patients with STEMI. Clearance of iohexol was measured (mGFR) before discharge. We evaluated and compared the Cockcroft-Gault (CG), the Modification of Diet in Renal Disease (MDRD-IDMS), the Chronic Kidney Disease Epidemiology (CKD-EPI) and the Grubb relative cystatin C (rG-CystC) with GFR regarding correlation, bias, precision and accuracy (P30). Agreement between eGFR and mGFR to discriminate CKD was assessed by Cohen's $\kappa$ statistics.

Results: MDRD-IDMS and CKD-EPI demonstrated good performance to estimate GFR (correlation 0.78 vs $0.81 \%$, bias $-1.3 \%$ vs $1.5 \%$, precision 17.9 vs $17.1 \mathrm{~mL} / \mathrm{min} 1.73 \mathrm{~m}^{2}$ and $\mathrm{P} 3082.5 \%$ vs $82.5 \%$ for MDRD-IDMS vs CKD-EPI). CKD was best classified by CKD-EPI ( $\kappa$ 0.83). CG showed the worst performance (correlation $0.73 \%$, bias $-1 \%$ to $3 \%$, precision $22.5 \mathrm{~mL} / \mathrm{min} 1.73 \mathrm{~m}^{2}$ and P30 75\%). The rG-CystC formula had a marked bias of $-17.8 \%$ and significantly underestimated mGFR ( $p=0.03)$. At arrival, CKD-EPI and $\mathrm{rG}$-CystC had almost perfect agreement in CKD classification $(\kappa=0.87)$, whereas at discharge agreement was substantially lower $(\kappa=0.59)$ and showed a significant discrepancy in CKD classification $(p=0.02)$. Median cystatin $C$ concentration increased by $19 \%$.

Conclusions: In acute STEMI, CKD-EPI showed the best CKD-classification ability followed by MDRDIDMS, whereas CG performed the worst. STEMI altered the performance of the cystatin $C$ equation during the acute phase, suggesting that other factors might be involved in the rise of cystatin $\mathrm{C}$.

\section{Strengths and limitations of the study}

- One strength of the study is that it is the first study to validate the performance of the most widely used glomerular filtration rate (GFR) estimates compared to measured GFR in patients with ST-elevation myocardial infarction, during the index hospitalisation.

- Another strength is that this is a pure methodological study and the patients were their own controls.

- One obvious limitation of our study is the small sample size.

- The performance of GFR estimates in various subgroups, based on chronic kidney disease stage, age, weight and gender, was not possible.

- Another limitation is that our cystatin C assay was not standardised against the international cystatin C reference material, which was not commercially available, and systematic error in the measurement of cystatin $C$ may have occurred.

\section{INTRODUCTION}

On the basis of estimated glomerular filtration rate (eGFR), previous studies have shown a powerful relationship between the severity of renal dysfunction and poor outcomes in ST-elevation myocardial infarction (STEMI).${ }^{1-7}$ Although renal function is not always known at arrival of the patients with STEMI, it is of crucial importance for dose adjustment of drugs cleared by the kidneys and careful use of contrast media. ${ }^{8}$ As measurements of GFR cannot be used for routine clinical praxis, formulas to estimate GFR, based on creatinine, have been developed. The Cockcroft-Gault (CG), and the Modification of Diet in Renal Disease (MDRD $)^{9} 10$ are the most widely used formulas to estimate GFR. However, their performance significantly varies in populations not similar to the one from which the formulas were 
derived. ${ }^{8}$ During the last years, new formulas such as the Chronic Kidney Disease-Epidemiology Collaboration (CKD-EPI $)^{11}$ based on creatinine have emerged and challenged the older formulas due to their higher accuracy. Nevertheless, all creatinine based formulas share a main limitation, the unpredictable production and tubular secretion of creatinine in various individuals and populations.

The relative Grubb cystatin $\mathrm{C}$ formula (rG-CystC) ${ }^{12}$ has shown a high accuracy, comparable to MDRD, using just one variable, the cystatin $\mathrm{C}$ concentration, and has probably overcome many limitations of the creatinine based estimates in different populations. However, measurements of cystatin $\mathrm{C}$ have suffered from a lack of universal standardisation. Furthermore, it remains unclear if inflammation, myocardial necrosis and atherosclerosis affect cystatin C levels in patients with MI. ${ }^{13} 14$

Since significant disagreements in CKD classification between formulas have been proven in MI populations, overestimation of GFR by formulas may have led to an overdosing of antithrombotic drugs and contributed to the observed higher bleeding rate. ${ }^{8}$ Despite the crucial role of renal function in the management of patients with STEMI, GFR estimates have not been validated against mGFR in that population during the acute phase. Therefore, it is still unclear if their prognostic impact only depends on their accuracy to estimate GFR or the coefficients that are included in the formulas.

The objective of this study was to validate the performance of the most commonly used formulas for estimation of GFR against mGFR during the index hospitalisation in a population with STEMI.

\section{MATERIAL AND METHODS}

Design and Study Population: This was a single centre, methodological study including patients with STEMI treated with primary percutaneous coronary intervention (PPCI). Patients with known advanced renal failure (on dialysis), cardiogenic shock at arrival or known allergy to iodine were excluded. Between November 2011 and February 2013, 40 patients were successfully included.

\section{GFR, plasma creatinine and cystatin C measurements}

Blood samples were obtained via a direct venous puncture at the time of randomisation (before coronary angiography) and before discharge (between days 4 and 7 after admission). Plasma creatinine and cystatin $\mathrm{C}$ were analysed at the central laboratory of Östergötland County Council (at Linköping University Hospital and at Norrköping Hospital).

Furthermore, from patient records, we retrospectively collected high sensitivity troponin $\mathrm{T}$ that was routinely measured $6-8 \mathrm{~h}$ after admission as a marker of infarct size.

\section{Determination of GFR}

GFR was determined by measurement of the plasma clearance of iohexol, ${ }^{15}$ a non-radioactive radiographic contrast medium (Omnipaque $300 \mathrm{mg} \mathrm{I} / \mathrm{mL}$; Nycomed Amersham AB, Sweden) before discharge, soon after blood samples for creatinine and Cystatin C measurements were obtained. Four millilitres of iohexol were injected intravenously into an antecubital vein and clearance was calculated from the remaining iohexol concentration in two plasma samples. The sampling time point was determined from eGFR, using MDRD- isotope dilution mass spectrometry (IDMS). If eGFR was $>40 \mathrm{~mL} /$ $\min / 1.73 \mathrm{~m}^{2}$, sampling was performed at 3 and $4 \mathrm{~h}$ after injection. For eGFR $<40 \mathrm{~mL} / \mathrm{min} / 1.73 \mathrm{~m}^{2}$, samples were drawn 6 and $8 \mathrm{~h}$ after injection. Plasma iohexol concentration was determined by high-performance liquid chromatography. The total coefficient of variation (CV) for the analysis method, during a 3-month period, was $4.0 \%$ for a control sample with an assigned value of $31 \mathrm{mg} / \mathrm{L}$ and $3.7 \%$ for a control sample with an assigned value of $62 \mathrm{mg} / \mathrm{L}(\mathrm{n}=56)$. For a person weighing $70 \mathrm{~kg}$ and sampling at $4 \mathrm{~h}$, these iohexol concentrations corresponded to GFR values of approximately 100 and $60 \mathrm{~mL} / \mathrm{min}$, respectively, without correction for body surface area (BSA). GFR was expressed in relative values: $\mathrm{mL} / \mathrm{min} / 1.73 \mathrm{~m}^{2}$ using the DuBois-DuBois formula for calculation of BSA.

\section{Determination of plasma creatinine}

Plasma creatinine was analysed by a kinetic alkaline picrate colometric method, a modification of the original method described by Jaffe using Advia 1650 and 1800 instruments (Siemens Healthcare Diagnostics). Precision measured during a 3-month period on four instruments showed a total $\mathrm{CV}$ of $4.3 \%$ at a creatinine level of $86 \mu \mathrm{mol} / \mathrm{L}$ and of $3.2 \%$ at a creatinine level of $380 \mathrm{\mu mol} / \mathrm{L}$ (reference values used: $60-105$ for adult males and 45-90 for adult females). Calibration is traceable to a primary reference material (SRM 967) with values assigned by IDMS and a zero-point calibrator was used.

\section{Measurement of cystatin C}

Plasma cystatin $\mathrm{C}$ was measured by an automated particle-enhanced immunoturbidimetric method on the Advia 1650 and 1800 analysis system (Siemens Healthcare Diagnostics) with reagents obtained from DakoCytomation and according to the procedure recommended by the reagent producer. Precision measured during a 3-month period on two instruments showed a total $\mathrm{CV}$ of $4.2 \%$ at a cystatin $\mathrm{C}$ concentration of $1.2 \mathrm{mg} / \mathrm{L}$ and $3.2 \%$ at $4.6 \mathrm{mg} / \mathrm{L}$ (reference values used, $0.55-1.15 \mathrm{mg} / \mathrm{L}$ for persons $1-50$ years of age and $0.63-1.44 \mathrm{mg} / \mathrm{L}$ for persons $>50$ years). All samples were analysed within 1 day after collection.

\section{Definition and staging of chronic kidney disease}

According to the National Kidney Foundation Kidney/ Disease Outcome Quality Initiative (NKF K/DOQI), CKD is defined as kidney damage persisting for more than 3 months. ${ }^{16}$ Patients with reduced kidney function 
should be classified into five CKD stages based on GFR. Patients in stage 1 (GFR $\geq 90 \mathrm{~mL} / \mathrm{min} / 1.73 \mathrm{~m}^{2}$, normal kidney function) and stage 2 (GFR $60-89 \mathrm{~mL} / \mathrm{min}$ / $1.73 \mathrm{~m}^{2}$, mild CKD) must have signs of kidney damage such as albuminuria or pathological imaging in order to be classified as CKD. Patients in stage 3 (GFR 30$59 \mathrm{~mL} / \mathrm{min} / 1.73 \mathrm{~m}^{2}$ ) and stage 4 (GFR $15-29 \mathrm{~mL} / \mathrm{min}$ / $1.73 \mathrm{~m}^{2}$ ) have moderate or severe CKD, respectively. Dialysis or GFR $<15 \mathrm{~mL} / \mathrm{min} / 1.73 \mathrm{~m}^{2}$ is defined as endstage renal failure (stage 5 ). In this article, from now on CKD is defined as CKD stages 3-5, that is, GFR less than $60 \mathrm{~mL} / \mathrm{min} / 1.73 \mathrm{~m}^{2}$.

\section{Statistical analysis and eGFR formulas}

The percentage of patients with CKD obtained with the four formulas and mGFR was compared with McNemar's test. Agreement between GFR estimates and between mGFR and eGFR regarding CKD classification to discriminate GFR greater and less than $60 \mathrm{~mL} / \mathrm{min}$ $1.73 \mathrm{~m}^{2}$ was assessed by Cohen's $\kappa$ statistics. A $\kappa$ value of 0.20 or less was considered as slight agreement; 0.210.40 as fair agreement; $0.41-0.60$ as moderate agreement; $0.61-0.80$ as substantial agreement; and $0.81-1.00$ as almost perfect agreement. ${ }^{17}$

Furthermore, we constructed Bland-Altman plots according to the original article by Bland and Altman. ${ }^{18}$ Since iohexol clearance was considered as the gold standard method, differences between eGFR and mGFR were plotted against that.

We assessed the diagnostic performance of the eGFR formulas compared to mGFR with respect to correlation, bias, precision and accuracy. ${ }^{19}$ Correlation between eGFR and mGFR was obtained from Pearson's correlation and reported as correlation coefficient (R). Bias was defined as the median percentage error between eGFR and mGFR; positive values indicate an overestimation of mGFR. A bias of less than $10 \%$ was considered as clinically acceptable. ${ }^{20}$ Precision was assessed as the IQR expressed in $\mathrm{mL} / \mathrm{min} / 1.73 \mathrm{~m}^{2}$ of the difference eGFR-mGFR. Accuracy within 30\% (P30) was the percentage of estimates within $30 \%$ of mGFR. A P30 of more than $75 \%$ is sufficient for clinical decisions. ${ }^{21}$ Differences in accuracy between $0 \%$ and $2 \%$ were regarded as equivalent accuracy, between $3 \%$ and $4 \%$ as slightly higher accuracy and $\geq 5 \%$ as significant higher accuracy. The $95 \%$ CI of P30 was calculated according to the formula:

$$
\begin{aligned}
95 \% \mathrm{CI}= & \mathrm{P} 30 \\
& \pm 1.96 \mathrm{x}\left[\mathrm{P} 30 \mathrm{x} \frac{(1-\mathrm{p} 30)}{\text { number of measurements }}\right]^{0.5} .
\end{aligned}
$$

We used the Ct CG, the MDRD-IDMS, the Chronic Kidney Disease-Epidemiology Collaboration CKD-EPI based on creatinine and the rG-CystC formulas to estimate GFR (see online supplementary material text box).
The $\mathrm{p}$ values $<0.05$ were considered statistically significant.

Data were analysed with the use of SPSS software, release V.21.0 (SPSS Inc.).

\section{Ethics}

The study protocol was approved by the regional ethical committee and all procedures were in accordance with the Helsinki Declaration concerning ethical principles for medical research involving human subjects and Good Clinical Practice Guidelines. All patients gave oral informed consent before inclusion and written informed consent after the PPCI.

\section{RESULTS}

Anthropometric and clinical characteristics are presented in table 1.

The median creatinine on arrival was $86 \mu \mathrm{mol} / \mathrm{L}$ and the median cystatin $\mathrm{C}$ was $1.1 \mathrm{mg} / \mathrm{L}$. Cystatin $\mathrm{C}$ increased by $19 \%$ during hospitalisation, whereas a $6 \%$ increase in creatinine value was observed (median values). Median values of measured and estimated (by each equation) GFR at admission and at discharge are presented in the online supplementary material table.

\section{CKD prevalence}

According to mGFR at discharge, $2.5 \%$ of patients had at least severe renal dysfunction, 30\% moderate renal dysfunction and $67.5 \%$ normal or mildly impaired renal function $(42.5 \%$ mild CKD and $25 \%$ normal renal function).

On arrival, the CG showed a tendency to overestimate the CKD prevalence compared to other formulas, but

\begin{tabular}{|c|c|}
\hline & Total \\
\hline Number & 40 \\
\hline Men, n (\%) & $29(73)$ \\
\hline Age, mean (SD) & $67(11)$ \\
\hline Weight, mean (SD) & $82(16)$ \\
\hline BMI, mean (SD) & $27(5)$ \\
\hline Active smoker, n (\%) & $13(33)$ \\
\hline Diabetes, n (\%) & $6(15)$ \\
\hline Hypertension, n (\%) & $18(45)$ \\
\hline Previous MI, n (\%) & $6(15)$ \\
\hline Previous PCI, n (\%) & $6(15)$ \\
\hline Previous stroke, n (\%) & $3(8)$ \\
\hline$\beta$ Blocker, n (\%) & $13(33)$ \\
\hline ACE/ARB inhibitor, $n(\%)$ & $14(35)$ \\
\hline $\begin{array}{l}\text { Creatinine (on arrival), } \\
\text { median (25-75th centile) }\end{array}$ & $86(74-95)$ \\
\hline $\begin{array}{l}\text { Cystatin C (on arrival), } \\
\text { median (25-75th centile) }\end{array}$ & $1.08(0.95-1.24)$ \\
\hline
\end{tabular}
no statistically significant differences could be detected. 
Table 2 Prevalence of moderate-severe CKD on arrival and at discharge

\begin{tabular}{|c|c|c|}
\hline & All & p Value* \\
\hline \multicolumn{3}{|l|}{ On arrival } \\
\hline $\mathrm{CG}$ & 35 (14) & \\
\hline MDRD-IDMS & $27.5(11)$ & \\
\hline CKD-EPI & 25 (10) & \\
\hline rG-CystC & 25 (10) & \\
\hline \multicolumn{3}{|l|}{ At discharge } \\
\hline CG & $42.5(17)$ & 0.3 \\
\hline MDRD-IDMS & $32.5(13)$ & 1.0 \\
\hline CKD-EPI & 30 (12) & 1.0 \\
\hline rG-CystC & 47.5 (19) & 0.03 \\
\hline lohexol & 32.5 (13) & \\
\hline \multicolumn{3}{|c|}{$\begin{array}{l}\text { *p Values for comparison of percentage of patients with } \\
\text { moderate-severe CKD obtained with the four formulas compared } \\
\text { to mGFR by using McNemar's test. } \\
\text { CG, Cockcroft-Gault; CKD-EPI, Chronic Kidney } \\
\text { Disease-Epidemiology Collaboration; mGFR, measured GFR; } \\
\text { MDRD-IDMS, Modification of Diet in Renal Disease-Isotope } \\
\text { Dilution Mass Spectrometry; rG-CystC, relative Grubb cystatin C. }\end{array}$} \\
\hline
\end{tabular}

The lowest prevalence of CKD was obtained when the CKD-EPI and the rG-CystC were used.

At discharge, the rG-CystC significantly overestimated the prevalence of CKD compared to mGFR, whereas the CG showed a similar tendency. The CKD prevalence according to the CKD-EPI and the MDRD-IDMS formulas were comparable to that obtained by mGFR (table 2).

The rG-CystC significantly overestimated the prevalence of CKD compared to the CKD-EPI $(p=0.02)$ and the MDRD $(\mathrm{p}=0.07)$.

The level of agreement between eGFR and mGFR to discriminate GFR $<60 \mathrm{~mL} / \mathrm{min} / 1.73 \mathrm{~m}^{2}$ is illustrated in table 3. At discharge, the CKD-EPI had an almost perfect agreement with mGFR according to Cohen's $\kappa$ value, followed by the MDRD-IDMS. The rG-CystC had a substantial agreement but was lower than the MDRD-IDMS, whereas the CG achieved the lowest agreement among the formulas tested (moderate agreement).

When agreement between different GFR estimates, both on arrival and at discharge, was examined (table 3), the creatinine-based formulas had an essentially unchanged agreement to each other, whereas rG-CystC showed an almost perfect agreement with MDRD-IDMS and CKD-EPI on arrival but a substantially lower agreement at discharge formulas.
The Bland and Altman plots for eGFR at discharge

The plots show that the magnitude of the differences did not systematically vary over the range of mGFR. All the creatinine based formulas had a tendency to overestimate the mGFR at the lower range of mGFR. Furthermore, the rG-CystC underestimated the renal function over the whole range of mGFR (figure 1).

\section{Correlation, bias, precision and accuracy}

The MDRD-IDMS, the CKD-EPI and the rG-CystC estimates had better correlation to mGFR, compared to the CG. The rG-CystC yielded the best correlation with mGFR. All creatinine-based estimates showed a low bias. The rG-CystC formula had a marked bias of $-17.8 \%$. The rG-CystC had the highest precision, followed by the CKD-EPI and the MDRD-IDMS that yielded an almost equal value, whereas the CG showed the lowest precision between the formulas tested. The CKD-EPI and the MDRD-IDMS had the highest accuracy. The rG-CystC had a slightly lower accuracy compared with the CKD-EPI and the MDRD-IDMS. The CG showed a considerably lower accuracy compared to other equations (table 4).

We found a moderate correlation between the cystatin $\mathrm{C}$ rise during hospitalisation (absolute values) and high sensitivity troponin values $6-8 \mathrm{~h}$ after admission $(\mathrm{R}=0.51$, $\mathrm{p}=0.01$ ) (see the online supplementary material figure).

\section{DISCUSSION}

This is, to the best of our knowledge, the first study to validate the performance of the most widely used GFR estimates compared to mGFR in patients with STEMI, during the index hospitalisation.

On the basis of mGFR, one-third of our patients had at least moderate renal dysfunction, but only a small minority had CKD 4-5. Large observational studies based on the GRACE and SWEDEHEART registries have shown similar results with $28.6 \%$ of patients with STEMI suffering from moderate CKD but only $6.4 \%$ from severe renal dysfunction $4 .^{22}$

At discharge, the MDRD-IDMS and the CKD-EPI formula had a good overall performance to estimate GFR. The two formulas showed a comparable and acceptable correlation, bias, precision and accuracy in this cohort with STEMI. The CKD-EPI had the best CKD-classification ability and an almost perfect

Table 3 Agreement between different estimates and mGFR to discriminate GFR greater and less than $60 \mathrm{~mL} / \mathrm{min} / 1.73 \mathrm{~m}{ }^{2}$

\begin{tabular}{llllll}
\hline & CG & MDRD-IDMS & CKD-EPI & rG-CystC & Iohexol \\
\hline CG & $+++/+++$ & $0.71 / 0.68$ & $0.65 / 0.74$ & $0.58 / 0.60$ & $+++/ 0.58$ \\
MDRD-IDMS & $0.71 / 0.68$ & $+++/+++$ & $0.94 / 0.94$ & $0.81 / 0.59$ & $+++/ 0.77$ \\
CKD-EPI & $0.65 / 0.74$ & $0.94 / 0.94$ & $+++/+++$ & $0.87 / 0.64$ & $+++/ 0.83$ \\
rG-CystC & $0.58 / 0.60$ & $0.81 / 0.59$ & $0.87 / 0.64$ & ++++++ & $+++/ 0.70$ \\
\hline
\end{tabular}

Cohen's $\kappa$ values for comparison at arrival/discharge.+++comparison was not performed.

CG, Cockcroft-Gault; CKD-EPI, Chronic Kidney Disease-Epidemiology Collaboration; GFR, glomerular filtration rate; mGFR, measured GFR; MDRD-IDMS, Modification of Diet in Renal Disease-Isotope Dilution Mass Spectrometry; rG-CystC, relative Grubb cystatin C. 

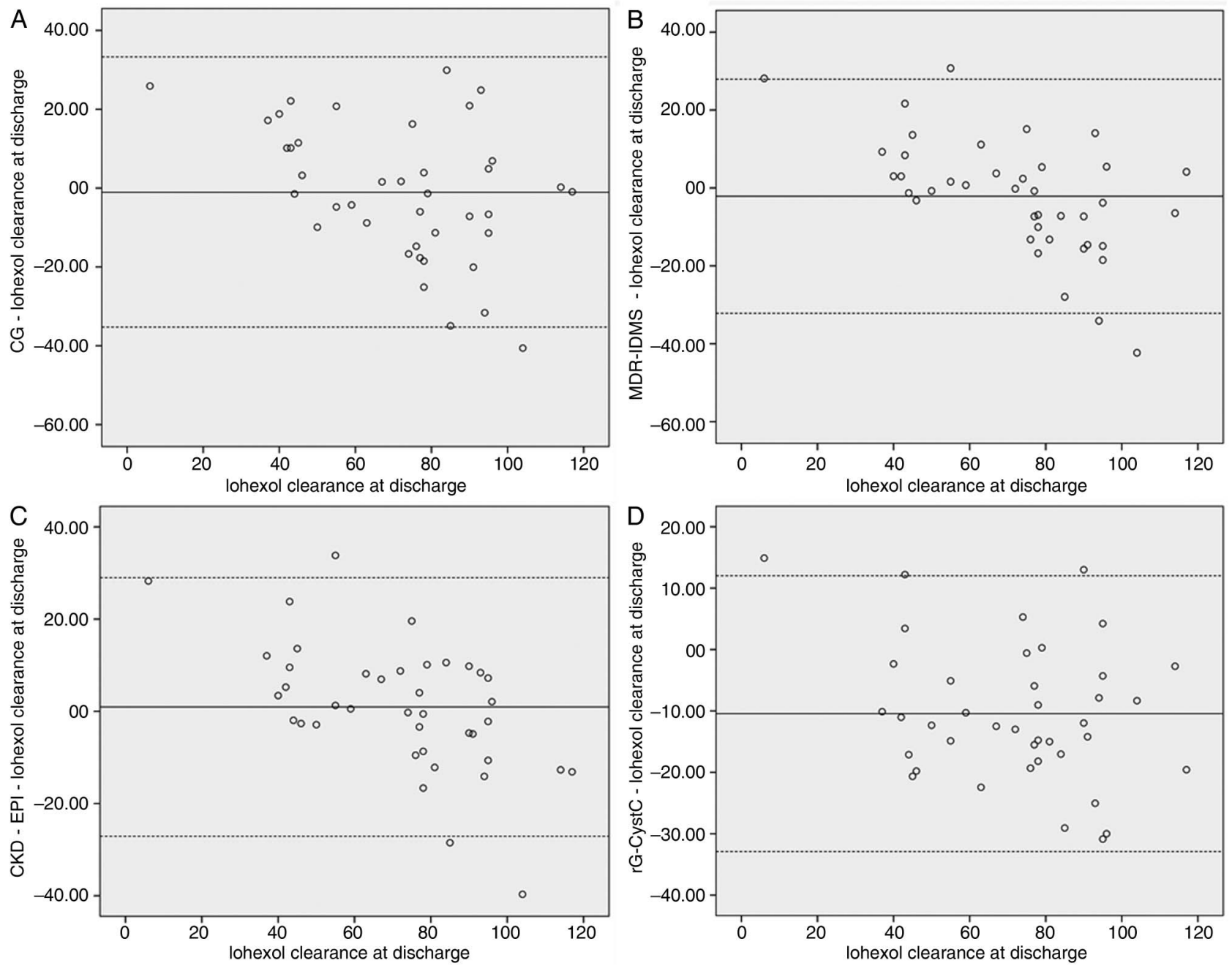

Figure 1 (A-D) Bland and Altman analysis. The CG (A), the MDRD-IDMS (B) the CKD-EPI (C) and the rG-CystC (D) formulas were used. The difference between estimated glomerular filtration rate (GFR) and measured GFR is presented on the $x$ axis. Measured GFR (mGFR) by iohexol clearance on the y axis. The continuous line represents the mean difference between eGFR and mGFR, whereas the dashed lines represent the limits of agreement ( \pm 2 SD of the difference). All values are expressed as relative GFR (mL/min/1.73 $\left.\mathrm{m}^{2}\right)$.

agreement with the mGFR and classified fewer patients as having CKD compared to other formulas. The CG formula showed the worst performance, with the lowest correlation, precision and accuracy, and a tendency to overestimate the prevalence of CKD compared to other creatinine-based.
Despite the fact that the rG-CystC formula showed a good overall accuracy, just slightly lower than the CKD-EPI and the MDRD-IDMS, the formula had a clinically unacceptable marked bias of $-17.8 \%$, due to a systematic underestimation of mGFR in almost the whole population. That resulted in a statistically significant

Table 4 Correlation, bias, precision and accuracy $(\mathrm{P} 30)$ of prediction equations to estimate relative $\mathrm{mGFR}\left(\mathrm{mL} / \mathrm{min} / 1.73 \mathrm{~m}^{2}\right)$

\begin{tabular}{lllll}
\hline At discharge & Correlation (R) & Bias, median error (\%) & $\begin{array}{l}\text { Precision (IQR), } \\
\mathbf{m L} / \mathbf{m i n} / \mathbf{1 . 7 3} \mathbf{~ m}^{\mathbf{2}}\end{array}$ & $\mathbf{P 3 0}$ (95\% Cl) \\
\hline CG & 0.73 & $-1.2(-1.3)$ & 22.5 & $75.0 \%(62 \%$ to $88 \%)$ \\
MDRD-IDMS & 0.78 & $-0.8(-1.3)$ & 17.9 & $82.5 \%(70.5 \%$ to $94.5 \%)$ \\
CKD-EPI & 0.81 & $0.9(1.5)$ & 17.1 & $82.5 \%(70.5 \%$ to $94.5 \%)$ \\
rG-CystC & 0.89 & $-12.2(-17.8)$ & 14.8 & $80.0 \%(68 \%$ to $92 \%)$ \\
\hline
\end{tabular}

Bias was defined as the median percentage error between eGFR and mGFR; positive values indicate an overestimation of mGFR. Precision was assessed as the IQR expressed in $\mathrm{mL} / \mathrm{min} / 1.73 \mathrm{~m}^{2}$ of the difference eGFR-mGFR. Accuracy within $30 \%$ (P30) was the percentage of estimates within $30 \%$ of mGFR. Correlation between eGFR and mGFR was reported as correlation coefficients (R).

CG, Cockcroft-Gault; CKD-EPI, Chronic Kidney Disease-Epidemiology Collaboration; eGFR, estimated glomerular filtration rate; mGFR, measured GFR; MDRD-IDMS, Modification of Diet in Renal Disease-Isotope Dilution Mass Spectrometry; rG-CystC, relative Grubb cystatin C. 
overestimation of the prevalence of CKD compared to mGFR and to other formulas, with a prevalence of $47.5 \%$ with rG-CystC compared to $32 . \%$ according to mGFR ( $p=0.03$ ).

Validation of the most widely used creatinine based GFR estimates, in the acute phase after a STEMI, showed a largely confirmatory result. The similar overall accuracy of the MDRD-IDMS and the CKD-EPI equations was consistent with the original CKD-EPI external validation cohort, where P30 was $84 \%$ for the CKD-EPI formula and $81 \%$ for the MDRD-IDMS formula. ${ }^{11}$ The low bias for the MDRD-IDMS and the CKD-EPI was comparable with the results in previous studies. ${ }^{23}$

The CKD-EPI showed the best ability to discriminate patients with CKD and that can be explained by the population studied. The CKD-EPI performs better in patients with GFR $\geq 60 \mathrm{~mL} / \mathrm{min} / 1.73 \mathrm{~m}^{2}$ and reduces the rate of a false-positive diagnosis of CKD (eGFR $\left.<60 \mathrm{~mL} / \mathrm{min} / 1.73 \mathrm{~m}^{2}\right) .{ }^{11}$ The original MDRD formula was developed by studying 1628 patients with nondiabetic CKD using the Jaffe method of creatinine measurement, but was re-expressed for use with the standardised serum creatine assay. ${ }^{24}$ The best accuracy is obtained in patients with CKD, whereas the formula has a tendency to underestimate the mGFR in patients without CKD. ${ }^{10}$

As in previous studies, ${ }^{8} 2526$ the CG formula had a tendency to overestimate the prevalence of CKD compared to other GFR estimates. The CG formula has many limitations. First, it was derived from a small population, predominantly men, and an arbitrary correction for women was proposed. Second, it calculates creatinine clearance that may significantly differ from GFR. Third, the formula has not been re-expressed for use with the standardised serum creatinine assay. However, the CG has been extensively used and previous studies have shown that the eGFR based on CG is a better predictor of adverse outcomes than that based on the MDRD-IDMS and probably the CKD-EPI formulas in patients with myocardial infarction. ${ }^{25-27}$ According to our results, the predictive ability of the CG formula should be attributed to the coefficients included in the formula and not to the better estimation of GFR, as other authors have previously suggested..$^{25}$

Validation of the rG-CystC formula in our population with STEMI showed inconsistent results with previous studies. In the development and validation population, the rG-CystC formula showed a very good performance, at least as good as the MDRD-IDMS one. ${ }^{12} 28$

In the absence of a universal calibrator for cystatin $\mathrm{C}$, the choice of the cystatin $\mathrm{C}$ measurement method is of crucial importance ${ }^{29}$ and can importantly affect the performance of a cystatin C-based equation that is not derived by using the same calibrator and method, resulting in a significant methodological error in GFR estimations. $^{30}$

In our study, we calculated eGFR by using a rG-CystC formula that was recommended by the manufacturer.
Therefore, an important methodological error in the cystatin $\mathrm{C}$ measurements was considered improbable. It should be noticed that the performance of the rG-CystC equation altered during the study period. At arrival, CKD-EPI and rG-CystC showed an almost perfect agreement, classified almost the same patients as having CKD, but at discharge their agreement was substantially lower and they showed an important discrepancy in CKD classification that reached statistical significance. During hospitalisation, cystatin $\mathrm{C}$ increased by $19 \%$, disproportional to creatinine rise. Theoretically, that could be attributed to the early detection of renal injury by cystatin $\mathrm{C}$ and not by creatinine. Nevertheless, that can be ruled out as the rG-CystC considerably overestimated the CKD prevalence compared to mGFR and the CKD-EPI showed an almost perfect agreement with mGFR at discharge.

Our findings suggest that other pathophysiological events were probably involved in the rise of cystatin $\mathrm{C}$ and the observed bias of the rG-CystC, for example, plaque rapture, inflammation and myocardial necrosis. Cystatin $\mathrm{C}$ has been shown to be an independent predictor of mortality and MI in patients with stable angina, ACS and STEMI, ${ }^{61-33}$ and previous studies have suggested that cystatin $\mathrm{C}$ is not only a marker of renal function but is also correlated with atherosclerosis, inflammation, plaque vulnerability and rupture ${ }^{13}{ }^{14} \mathrm{We}$ examined a possible relationship between cystatin $\mathrm{C}$ increase and troponin level in our population. We found a significant but moderate correlation between cystatin $\mathrm{C}$ rise and troponin and a weaker correlation between creatinine rise and troponin. This partially reflects the cardiorenal syndrome but could also indicate a relationship between the infarct size and the cystatin $\mathrm{C}$ elevation. Apart from an elevated production of cystatin $\mathrm{C}$ during the acute phase of myocardial infarction, an altered glomerular endothelial permeability due to infarct-related inflammation may impair the filtration rate of cystatin $\mathrm{C}$ that is more than a 100 times larger molecule than creatinine, leading to an accumulation of cystatin $\mathrm{C}$ in the blood. ${ }^{34}$

However, this study was not designed to give any explanatory results but only to evaluate the performance of the most widely used eGFR formulas.

\section{CONCLUSION}

In the acute stage of STEMI, the performance of the MDRD-IDMS and the CKD-EPI was clinically acceptable. The CKD-EPI had the best CKD-classification ability and should probably be preferred. The rG-CystC equation showed a marked bias and our findings suggest that myocardial infarction alters the performance of the equation and other pathophysiological mechanisms are probably involved in the rise of cystatin C.

\section{Clinical implications}

According to our results, MDRD and CKD EPI should be the formulas used for estimation of GFR in the acute 
phase of STEMI as they showed a good overall performance. On the other hand, CG should be abandoned as it overestimates the prevalence of CKD as previously shown in patients with stable CAD. Finally, cystatin $\mathrm{C}$ based formulas should be used with caution in the acute phase of STEMI until more studies confirm or reject our findings.

Contributors DV and SSL are responsible for the conception and design of the study; they analysed and interpreted the data and drafted the manuscript. $\mathrm{JA}$ and ES are responsible for the conception and design of the study; they critically revised the manuscript and added some important intellectual content. MS critically revised the manuscript from a nephrologist's point of view and added some intellectual content.

Funding This research received no specific grant from any funding agency in the public commercial or not-for-profit sectors.

Competing interests None declared

Patient consent Obtained.

Ethics approval Linköping Ethical Review Board.

Provenance and peer review Not commissioned; externally peer reviewed.

Data sharing statement No additional data are available.

Open Access This is an Open Access article distributed in accordance with the Creative Commons Attribution Non Commercial (CC BY-NC 4.0) license, which permits others to distribute, remix, adapt, build upon this work noncommercially, and license their derivative works on different terms, provided the original work is properly cited and the use is non-commercial. See: http:// creativecommons.org/licenses/by-nc/4.0/

\section{REFERENCES}

1. Holzmann MJ, Ivert T, Jungner I, et al. Renal function assessed by two different formulas and incidence of myocardial infarction and death in middle-aged men and women. $J$ Intern Med 2010;267:357-69.

2. Bae EH, Lim SY, Cho KH, et al. GFR and cardiovascular outcomes after acute myocardial infarction: results from the Korea Acute Myocardial Infarction Registry. Am J Kidney Dis 2012;59:795-802.

3. Anavekar NS, McMurray JJ, Velazquez EJ, et al. Relation between renal dysfunction and cardiovascular outcomes after myocardial infarction. N Engl J Med 2004;351:1285-95.

4. Sederholm Lawesson S, Alfredsson J, Szummer K, et al. Prevalence and prognostic impact of chronic kidney disease in STEMI from a gender perspective: data from the SWEDEHEART register, a large Swedish prospective cohort. BMJ Open 2015;5:e008188.

5. Fox KA, Antman EM, Montalescot G, et al. The impact of renal dysfunction on outcomes in the ExTRACT-TIMI 25 trial. J Am Coll Cardiol 2007:49:2249-55.

6. Akgul O, Uyarel $\mathrm{H}$, Ergelen $\mathrm{M}$, et al. Predictive value of elevated cystatin $C$ in patients undergoing primary angioplasty for ST-elevation myocardial infarction. J Crit Care 2013;28:882.e13-20.

7. Orvin K, Eisen A, Goldenberg I, et al. The proxy of renal function that most accurately predicts short- and long-term outcome after acute coronary syndrome. Am Heart J 2015;169:702-712.e3.

8. Melloni C, Peterson ED, Chen AY, et al. Cockcroft-Gault versus modification of diet in renal disease: importance of glomerular filtration rate formula for classification of chronic kidney disease in patients with non-ST-segment elevation acute coronary syndromes. J Am Coll Cardiol 2008;51:991-6.

9. Cockcroft DW, Gault MH. Prediction of creatinine clearance from serum creatinine. Nephron 1976;16:31-41.

10. Levey AS, Coresh J, Greene T, et al. Chronic Kidney Disease Epidemiology Collaboration. Using standardized serum creatinine values in the modification of diet in renal disease study equation for estimating glomerular filtration rate. Ann Intern Med 2006;145:247-54.

11. Levey AS, Stevens LA Schmid $\mathrm{CH}$, et al. A new equation to estimate glomerular filtration rate. Ann Intern Med 2009;150:604-12.

12. Grubb A, Nyman U, Bjork J, et al. Simple cystatin C-based prediction equations for glomerular filtration rate compared with the modification of diet in renal disease prediction equation for adults and the Schwartz and the Counahan-Barratt prediction equations for children. Clin Chem 2005:51:1420-31.

13. Antoniadis AP, Chatzizisis YS, Giannoglou GD. Pathogenetic mechanisms of coronary ectasia. Int J Cardiol 2008;130:335-43.

14. Ge C, Ren F, Lu S, et al. Clinical prognostic significance of plasma cystatin $\mathrm{C}$ levels among patients with acute coronary syndrome. Clin Cardiol 2009;32:644-8.

15. O'Reilly PH, Brooman PJ, Martin PJ, et al. Accuracy and reproducibility of a new contrast clearance method for the determination of glomerula filtration rate. BrMed J (Clin Res Ed) 1986;293:234-6.

16. National Kidney Foundation. K/DOQI clinical practice guidelines for bone metabolism and disease in chronic kidney disease. $A m \mathrm{~J}$ Kidney Dis 2003;42(4 Suppl 3):S1-201.

17. Landis JR, Koch GG. The measurement of observer agreement for categorical data. Biometrics 1977;33:159-74.

18. Bland JM, Altman DG. Statistical methods for assessing agreement between two methods of clinical measurement. Lancet 1986;1:307-10.

19. National Kidney Foundation. K/DOQI clinical practice guidelines for chronic kidney disease: evaluation, classification, and stratification. Am J Kidney Dis 2002;39(2 Suppl 1):S1-266.

20. Spinler SA, Nawarskas JJ, Boyce EG, et al. Predictive performance of ten equations for estimating creatinine clearance in cardiac patients. Iohexol Cooperative Study Group. Ann Pharmacother 1998;32:1275-83.

21. Goolsby MJ. National Kidney Foundation Guidelines for chronic kidney disease: evaluation, classification, and stratification. $J A m$ Acad Nurse Pract 2002;14:238-42.

22. Moscucci M, Fox KA, Cannon CP, et al. Predictors of major bleeding in acute coronary syndromes: the Global Registry of Acute Coronary Events (GRACE). Eur Heart J 2003;24:1815-23.

23. Bjork J, Jones I, Nyman U, et al. Validation of the Lund-Malmo, Chronic Kidney Disease Epidemiology (CKD-EPI) and Modification of Diet in Renal Disease (MDRD) equations to estimate glomerular filtration rate in a large Swedish clinical population. Scand J Urol Nephrol 2012;46:212-22.

24. Levey AS, Coresh J, Greene T, et al., Chronic Kidney Disease Epidemiology Collaboration. Expressing the Modification of Diet in Renal Disease Study equation for estimating glomerular filtration rate with standardized serum creatinine values. Clin Chem 2007:53:766-72.

25. Szummer K, Lundman P, Jacobson SH, et al., SWEDEHEART. Cockcroft-Gault is better than the Modification of Diet in Renal Disease study formula at predicting outcome after a myocardial infarction: data from the Swedish Web-system for Enhancement and Development of Evidence-based care in Heart disease Evaluated According to Recommended Therapies (SWEDEHEART) Am Heart J 2010;159:979-86.

26. Tomaszuk-Kazberuk A, Kozuch M, Malyszko J, et al. Which method of GFR estimation has the best prognostic value in patients treated with primary PCI: Cockcroft-Gault formula, MDRD, or CKD-EPI equation?-A 6-year follow-up. Ren Fail 2011;33:983-9.

27. Kontos MC, Jamal SM, Ornato JP, et al. Comparison of the modification of diet in renal disease and the Cockcroft-Gault equations for predicting mortality in patients admitted for exclusion of myocardial ischemia. Am J Cardiol 2008;102:140-5.

28. Sterner G, Bjork J, Carlson J, et al. Validation of a new plasma cystatin C-based formula and the Modification of Diet in Renal Disease creatinine-based formula for determination of glomerular filtration rate. Scand J Urol Nephrol 2009;43:242-9.

29. Tidman M, Sjostrom P, Jones I. A Comparison of GFR estimating formulae based upon s-cystatin $C$ and s-creatinine and a combination of the two. Nephrol Dial Transplant 2008;23:154-60.

30. Delanaye P, Pieroni L, Abshoff C, et al. Analytical study of three cystatin $\mathrm{C}$ assays and their impact on cystatin C-based GFR-prediction equations. Clin Chim Acta 2008;398:118-24.

31. Akerblom A, Wallentin L, Siegbahn A, et al. Cystatin C and estimated glomerular filtration rate as predictors for adverse outcome in patients with ST-elevation and non-ST-elevation acute coronary syndromes: results from the Platelet Inhibition and Patient Outcomes study. Clin Chem 2012;58:190-9.

32. Silva D, Cortez-Dias N, Jorge C, et al. Cystatin C as prognostic biomarker in ST-segment elevation acute myocardial infarction. Am $J$ Cardiol 2012;109:1431-8.

33. Ichimoto E, Jo K, Kobayashi $\mathrm{Y}$, et al. Prognostic significance of cystatin $\mathrm{C}$ in patients with ST-elevation myocardial infarction. Circ $J$ 2009;73:1669-73.

34. Park M, Vittinghoff E, Ganz P, et al. Role of soluble endothelia cell-selective adhesion molecule biomarker in albuminuria and kidney function changes in patients with coronary artery disease: the Heart and Soul Study. Arterioscler Thromb Vasc Biol 2014;34:231-6. 